\title{
Ghrelina e Secretagogos do Hormônio de Crescimento (GHS): Modulação da Secreção do Hormônio de Crescimento e Perspectivas Terapêuticas
}

revisão

\author{
SILVIa R. CoRrea-SILVA \\ larissa Bianca PaIVa Cunha de Sá \\ Ana-Maria J. Lengyel
}

Disciplina de Endocrinologia da Escola Paulista de Medicina da Universidade Federal de São Paulo, SP, Brasil.

A secreção do hormônio de crescimento $(\mathrm{GH})$ é modulada pelo hormônio liberador de hormônio de crescimento $(\mathrm{GHRH})$ e pela somatostatina. $\mathrm{Na}$ última década foi descoberto um terceiro mecanismo de controle, envolvendo os secretagogos de GH (GHS). A ghrelina, o ligante endógeno do receptor dos GHS, é um peptídeo acilado produzido no estômago, que também é sintetizado no hipotálamo. Este peptídeo é capaz de liberar $\mathrm{GH}$, além de aumentar a ingesta alimentar. A ghrelina endógena parece amplificar o padrão básico de secreção de $\mathrm{GH}$, ampliando a resposta do somatotrofo ao GHRH, estimulando múltiplas vias intracelulares interdependentes. Entretanto, seu local de atuação predominante é o hipotálamo. Neste trabalho, será apresentada revisão sobre a descoberta da ghrelina, os mecanismos de ação e o possível papel fisiológico dos GHS e da ghrelina na secreção de $\mathrm{GH}$ e, finalmente, as possíveis aplicações terapêuticas destes compostos. (Arq Bras Endocrinol Metab 2008; 52/5:726-733)

Descritores: Ghrelina; GH; Secretagogos do hormônio de crescimento; GHRP-6

\begin{abstract}
Ghrelin and Growth Hormone Secretagogues (GHS): Modulation of Growth Hormone Secretion and Therapeutic Applications.

Growth hormone-releasing hormone (GHRH) and somatostatin modulate growth hormone $(\mathrm{GH})$ secretion. A third mechanism was discovered in the last decade, involving the action of growth hormone secretagogues (GHS). Ghrelin, the endogenous ligand of the GHS-receptor, is an acylated peptide mainly produced by the stomach, but also synthesized in the hypothalamus. This compound increases both $\mathrm{GH}$ release and food intake. Endogenous ghrelin might amplify the basic pattern of GH secretion, optimizing somatotroph responsiveness to $\mathrm{GHRH}$, activating multiple interdependent intracellular pathways. However, its main site of action is the hypothalamus. In the current paper it is reviewed the available data on the discovery of this peptide, the mechanisms of action and possible physiological roles of the GHS and ghrelin on GH secretion, and finally, the possible therapeutic applications of these compounds. (Arq Bras Endocrinol Metab 2008; 52/5:726-733)
\end{abstract}

Keywords: Ghrelin; GH; Growth hormone secretagogues; GHRP-6

\section{INTRODUÇÃO}

Recebido em 25/5/2008

Aceito em 30/5/2008
E M 1977, ANTES DA IDENTIFICAÇÃO do hormônio liberador do hormônio de Crescimento (GHRH), Bowers e cols. (1) descobriram um novo grupo de substâncias sintéticas com capacidade de liberar hormônio de crescimento (GH) (2). Estes compostos foram desenvolvidos a partir da molécula de 
metencefalina e, inicialmente, estimulavam a liberação de GH de modo pouco eficaz. Modificações químicas posteriores levaram ao desenvolvimento de substâncias mais potentes, como GHRP-6, GHRP-2, hexarelina e MK-0677 (2). Na última década, vários estudos foram realizados com estes secretagogos de GH (GHS), especialmente com GHRP-6, e os dados obtidos sugeriram possível papel destes compostos na modulação da secreção de GH (3). Foi demonstrado que os GHS liberam GH por mecanismos diferentes daqueles ativados pelo GHRH. Além disso, eles agem por meio de receptores diferentes daqueles do GHRH, por receptores próprios. Em 1996, Howard e cols. (4) clonaram o receptor de GHS (GHS-R), que se localiza principalmente na hipófise anterior e no hipotálamo. Em 1999, Kojima e cols. (5) descobriram, no estômago, o ligante endógeno deste receptor até então órfão, e este novo hormônio foi chamado ghrelina. A ghrelina é encontrada em pequenas quantidades no hipotálamo e induz a liberação de GH de modo muito potente $(5,6)$. Este hormônio é um novo membro da família de peptídeos cérebro-intestinais, e também está envolvido no controle do apetite, efeito aparentemente independente da liberação de $\mathrm{GH}(7,8)$. Outras possíveis ações da ghrelina estão sendo atualmente investigadas.

\section{RECEPTOR DOS SECRETAGOGOS DO HORMÔNIO DE CRESCIMENTO}

Em 1996, Howard e cols. (4) clonaram o GHS-R, que pertence à família de receptores acoplados à proteína $\mathrm{G}$, com sete domínios transmembranas e três alças intra e extracelulares. Existem dois subtipos de receptores, o GHS-Rla, que é ativo, e o GHS-Rlb, uma isoforma menor, que aparentemente não tem atividade biológica (4). A existência de outros subtipos é bastante provável. O GHS-Rla humano tem 366 aminoácidos e é altamente conservado na evolução. Este receptor está presente em vários tecidos, incluindo a hipófise anterior e o hipotálamo, e em outras áreas do cérebro, como hipocampo e substância negra. Em virtude de sua localização, tem sido sugerido que o GHS-R possa modular ritmos biológicos, humor, memória, aprendizado e apetite (2). Na hipófise, o GHS-R foi encontrado exclusivamente nos somatotrofos, por meio de imunoistoquímica (2). Em camundongos knockout para o GHS-Rla, a ghrelina não é capaz de aumentar a liberação de GH ou a ingesta alimentar, o que indica que este tipo de receptor esteja envolvido em ambas as ações da ghrelina (9). O GHS-Rla está presente em outros tecidos, como pâncreas, coração, adrenal e tireóide. $\mathrm{O}$ GHS-Rlb tem distribuição ampla no organismo, mas a sua função ainda é desconhecida (10).

\section{GHRELINA}

Em 1999, Kojima e cols. isolaram, a partir de extratos de estômago, um peptídeo de 28 aminoácidos que possuía modificação (ácido n-octanoil, um ácido graxo) no terceiro resíduo, que é uma serina (5). Este composto hidrofóbico, que é o primeiro peptídeo bioativo natural modificado por um ácido acil, foi chamado ghrelina. A ghrelina e os GHS, como o GHRP-6, não têm similaridade estrutural. O resíduo n-octanoil é essencial para algumas de suas atividades biológicas, incluindo a liberação de GH e a estimulação do apetite. Os fragmentos menores, com os primeiros quatro a cinco resíduos, com a serina acilada intacta são também capazes de ativar a transdução de sinal de GHS Rla in vitro (7). A principal forma circulante, a ghrelina não-acilada, pode ter ações não-endócrinas (5). O local de maior produção de ghrelina é o estômago e seus níveis são reduzidos em $80 \%$ após a gastrectomia ou o bypass gástrico em humanos (11). A ghrelina acilada atravessa a barreira hematoencefálica, e este transporte ocorre em ambas as direções (12). O gene que codifica a ghrelina está localizado no cromossomo $3 \mathrm{em}$ homens (5). Este gene codifica um precursor de 117 aminoácidos, com homologia de $82 \%$ entre as espécies (5). No estômago, as duas isoformas de RNAm de prepro-ghrelina são produzidas pelo mesmo gene por splicing alternativo. Um codifica o precursor da ghrelina, o outro codifica o precursor da des-Gln 14 ghrelina, que não tem a glutamina na posição 145. Este peptídeo tem 27 aminoácidos e é biologicamente ativo, mas está presente em pequenas quantidades no organismo. A ghrelina é encontrada na camada submucosa no fundo do estômago, nas células oxínticas endócrinas (X/A) que estão perto dos capilares e sem contato com o lúmen. Este hormônio também é encontrado, em concentrações menores, em outras regiões do trato gastrintestinal (7). Tanto a ghrelina quanto seu RNAm estão presentes no núcleo arqueado do hipotálamo e na hipófise $(5,10)$. Na hipófise, a ghrelina poderia agir de modo autócrino ou parácrino. A ghrelina é capaz de modular a transcrição de Pitl, e é expressa nos lactotrofos, somatotrofos e tireotrofos, células que são dependentes da expressão do gene Pitl para diferenciação (8). Nos tecidos periféri- 
cos, a ghrelina tem distribuição ampla, e é encontrada no rim, na placenta, no pulmão, nos ovários e nos testículos, mas seu papel nestes tecidos ainda não foi esclarecido (10). O receptor biologicamente ativo (GHS Rla) não está presente nos mesmos locais nos quais a ghrelina é encontrada, portanto é provável que existam outros subtipos de receptor (10). Quantidades consideráveis de ghrelina estão presentes na circulação e este peptídeo tem várias ações além da regulação do $\mathrm{GH}$ $(7,8)$. A ghrelina aumenta a ingestão alimentar pela ativação dos neurônios NPY/AgRP (proteína relacionada ao agouti) no hipotálamo, efeito oposto ao da leptina $(7,8)$. A ghrelina promove aumento da liberação de $\mathrm{GH}$ em animais e em homens, e também induz a secreção de prolactina, ACTH, cortisol e aldosterona in vivo $(5,6,13,14)$. Este hormônio causa aumento discreto da glicemia e redução dos níveis circulantes de insulina (14). A descoberta da ghrelina reforça a idéia da existência de uma terceira via de regulação da secreção de $\mathrm{GH}$, cujo papel fisiológico ainda permanece indeterminado $(2,3,7,8)$.

\section{MECANISMO DE AÇÃO E PAPEL FISIOLÓGICO DA GHRELINA E DOS GHS NA LIBERAÇÃO DE GH}

Os GHS e a ghrelina agem tanto no hipotálamo quanto na hipófise para modular a secreção de $\mathrm{GH}(3,7,8)$. Estes peptídeos são capazes de ativar diretamente os GHS-R nas células hipofisárias in vitro (5). Quando o GHRH é associado aos GHS ou à ghrelina in vitro, uma resposta aditiva é observada na maioria dos estudos. Entretanto, quando estes peptídeos são administrados junto com o GHRH in vivo, é observado efeito sinérgico na liberação de $\mathrm{GH}$, o que indica mecanismos de ação diferentes dos GHS e do GHRH, e sugere que o principal sítio de ação dos GHS seja o hipotálamo (6,14-16). Além disso, na desconexão hipotálamohipofisária há diminuição acentuada da liberação de GH após GHRP-6 ou ghrelina, tanto em animais quanto em homens $(17,18)$. Foi demonstrado que é necessário um sistema GHRH intacto para que estas ações possam ocorrer. A administração de anticorpos contra o GHRH diminui a pulsatilidade do GH e a resposta do GH à ghrelina e aos GHS em ratos (19). A liberação de GH induzida por GHS é também inibida por um antagonista do GHRH (20). No camundongo lit/lit, que tem mutação no receptor de GHRH, os GHS não aumentam a liberação de $\mathrm{GH}$, mas há aumento da ex- pressão de $c$-fos hipotalâmico, que é um marcador de atividade neuronal (21). A resposta do $\mathrm{GH}$ ao GHS está diminuída em humanos com mutação no receptor de GHRH, mas os efeitos liberadores de ACTH e prolactina estão preservados, sugerindo que estes últimos são mediados pelo hipotálamo (22). O núcleo arqueado é o alvo principal da ação da ghrelina, onde ela é capaz de se ligar e ativar o GHS-R. Foi demonstrado que os GHS e a ghrelina agem centralmente, aumentando a atividade elétrica e a expressão de $c$-fos em uma subpopulação de células no núcleo arqueado, algumas das quais são neurônios produtores de GHRH (21). Além disso, um quarto destes neurônios expressam o GHS-R, sugerindo efeito direto do GHS nestas células (19) (Figura 1). A ghrelina também promove a liberação de GHRH de tecido hipotalâmico in vitro, mas isso não é observado com os GHS $(23,24)$. Contudo, os GHS aumentam a liberação de GHRH no sistema porta-hipofisário de ovelhas (25). Os GHS e a ghrelina não alteram a secreção de somatostatina hipotalâmica na maioria dos estudos in vivo e in vitro (23-25). Entretanto, os GHS agem como antagonistas funcionais da somatostatina. Eles causam despolarização do somatotrofo e aumentam o número de células secretoras de $\mathrm{GH}$, enquanto a somatostatina tem efeito contrário (26).

Foi proposto um modelo de ação dos GHS/ghrelina, que envolve: 1) antagonismo funcional da somatostatina; 2) ativação dos neurônios produtores de GHRH no núcleo arqueado, levando ao aumento da

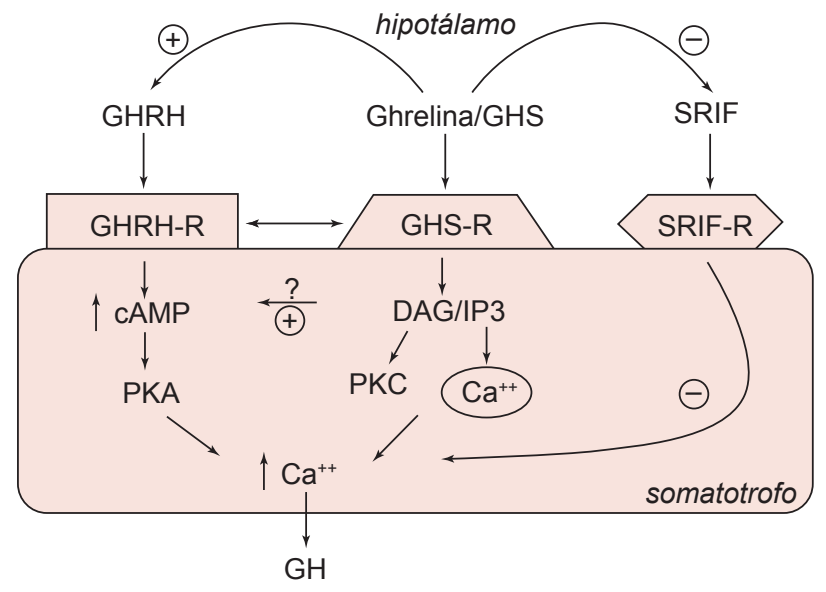

$\mathrm{PKA}=$ proteína quinase $\mathrm{A} ; \mathrm{PKC}=$ proteína quinase $\mathrm{C}$.

Figura 1. Modelo proposto para as interações entre o $\mathrm{GHRH}$ e a ghrelina/GHS em nível hipotalâmico e hipofisário. 
liberação de GHRH; 3) amplificação do efeito do GHRH no somatotrofo (2). Na hipófise, os GHS/ ghrelina e o GHRH se ligam a receptores diferentes, e há evidência de cross-talk entre os dois receptores (7). Estes peptídeos também ativam diferentes vias de transdução intracelular em nível somatotrófico. $\mathrm{O}$ GHRH estimula o AMPc intracelular e os mecanismos dependentes da proteína quinase $\mathrm{A}$, enquanto o GHRP-6 ativa a proteína quinase $\mathrm{C}$, via inositol trifosfato, com aumento nas concentrações de cálcio intracelular $(2,4)$. Foi demonstrado, recentemente, em células hipofisárias de suínos e babuínos que a ghrelina é capaz de estimular múltiplas vias de sinalização intracelular independentes, envolvendo proteína quinase A, C, MAPK, óxido nítrico/cGMP e sistemas de cálcio extracelular, com efeito mais amplo que a maioria dos GHS $(27,28)$.

O papel da ghrelina circulante na secreção de GH é bastante controverso. No rato, a secreção de ghrelina ocorre de modo pulsátil, sem correlação com pulsos de GH, mas em associação com ciclos de alimentação e sono (29). Também em ratos, os níveis de ghrelina circulante não se alteram com variações dos níveis de $\mathrm{GH}$ (30). A imunoneutralização da ghrelina não modifica a pulsatilidade de $\mathrm{GH}$, enquanto anticorpos anti-GHRH bloqueiam completamente a liberação pulsátil de $\mathrm{GH}$ endógeno (31). Em humanos, a administração de um antagonista do GHRH inibe acentuadamente a secreção de GH nas 24 horas, mas não afeta os níveis circulantes de ghrelina (32). Entretanto, em ratos, a administração intracerebroventricular ou periférica de antagonistas do GHS-Rla diminui a secreção espontânea de $\mathrm{GH}$, com redução da amplitude dos pulsos e dos níveis médios de GH (33-35). Uma mutação missense no GHS-R, que prejudica gravemente a ligação da ghrelina, foi associada a um caso de baixa estatura familiar (36). Em voluntários saudáveis foi mostrado que a ghrelina circulante está relacionada aos pulsos de $\mathrm{GH}$, sugerindo que ela participa da modulação da secreção pulsátil de GH ou que os dois hormônios são regulados em paralelo (37). Existem dados sugerindo que a ghrelina endógena poderia amplificar o padrão básico de secreção de GH (33-35), pela otimização da responsividade do somatotrofo ao GHRH (38). Todavia, estudos com animais knockout para a ghrelina não mostraram efeito importante na regulação da secreção de $\mathrm{GH}$ (39). Ao contrário do previsto, estes animais não eram anões anoréticos. Entretanto, níveis diminuídos de GH e
IGF-1 foram observados em modelos transgênicos com expressão reduzida do RNAm do GHS-R no núcleo arqueado (40). Também, camundongos knockout para GHS-R apresentavam baixo peso e diminuição dos níveis de IGF-l (9). É possível que os efeitos da ghrelina na secreção de GH possam se tornar relevantes durante estados de balanço energético negativo (30). Contudo, estudos adicionais são necessários para elucidar o papel fisiológico da ghrelina/GHS na secreção de GH.

\section{REGULAÇÃo DA SECREÇÃo DE GH PELO GHRP-6 E PELA GHRELINA EM HUMANOS}

O GHRP-6 e a ghrelina aumentam a liberação de GH de modo dose-dependente, in vivo e in vitro, em várias espécies, inclusive no homem $(1,5,6,13,16)$. A ghrelina é capaz de liberar GH in vitro de modo semelhante ao GHRH (5). Entretanto, in vivo, a administração endovenosa de ghrelina, na dose de $1 \mu \mathrm{g} / \mathrm{kg}$, aumenta a liberação de $\mathrm{GH}$ de modo potente e esta resposta é maior que a obtida com o GHRH, a hexarelina e o GHRP-6 $(13,14,41)$. Este efeito não é específico já que também há aumento de prolactina, ACTH, cortisol e aldosterona (14), discreta elevação de glicemia e queda nos níveis de insulina (14). Quando a ghrelina ou o GHRP-6 são administrados em conjunto com o GHRH ocorre efeito sinérgico, que é mais bem observado com baixas doses de ghrelina $(0,08 \mu \mathrm{g} / \mathrm{kg}$ e $0,2 \mu \mathrm{g} / \mathrm{kg})(14,16)$. A administração combinada de GHRP-6 e GHRH é atualmente excelente teste para o diagnóstico de deficiência de GH em adultos. Não existem diferenças na responsividade do GH ao GHRP-6 e à ghrelina quanto ao sexo, mas há diminuição da resposta relacionada à idade $(3,15,42)$. Indivíduos obesos têm diminuição da resposta do GH ao GHRP-6 e à ghrelina $(43,44)$. Há grande reprodutibilidade das respostas dos GHS em indivíduos normais estudados em ocasiões diversas, diferente do observado para o GHRH. Fatores como hiperglicemia, ácidos graxos livres e somatostatina diminuem a resposta de GH induzida pelo GHRP-6 e pela ghrelina $(45,46)$. Os efeitos da ghrelina e do GHRP-6 sobre a liberação de GH em diversas endocrinopatias, como doença de Cushing, disfunções tireoidianas, entre outras, foram por nós extensamente investigados e os dados mais relevantes, bem como os resultados de literatura, estão em revisões recentes sobre esse tema $(47,48)$. 


\section{PERSPECTIVAS TERAPÊUTICAS DOS GHS/GHRELINA}

O potencial terapêutico de vários análogos sintéticos dos GHS vem sendo amplamente estudado nos últimos anos. O maior enfoque tem sido a possibilidade de uso destes compostos, especialmente os ativos por via oral, como alternativa para o tratamento da deficiência de GH. A administração de uma única dose do análogo MK-0677, por via oral, em adultos com deficiência de GH idiopática desde a infância, leva ao aumento significante da secreção de GH e de IGF-1 (49). O MK-0677 também foi utilizado em crianças com deficiência de GH por oito dias, e também houve aumento significativo de GH, IGF-1 e IGFBP-3 em algumas crianças (50). Mais recentemente outro agonista oral dos GHS, o NN703, foi utilizado em 83 pacientes adultos com deficiência de GH durante seis dias, e apenas 11\% atingiram pico de $\mathrm{GH}$ maior que $5 \mu \mathrm{g} / \mathrm{L}$ após sua administração (51). A administração intranasal de GHRP-2, outro GHS, em crianças com baixa estatura (50\% com deficiência de GH e 50\% com baixa estatura idiopática) durante três meses levou a pequeno aumento, mas significante, da velocidade de crescimento, sem mudanças nas concentrações de IGF-1 e de IGFBP-3 ou nos picos de GH (52). Embora o tratamento com os GHS possa ser mais fisiológico, uma vez que esses compostos promovem a liberação endógena de $\mathrm{GH}$, os estudos não mostraram benefício adicional de seu uso em relação ao tratamento com GH. A vantagem adicional do uso dos GHS seria a administração oral, já que o GH é administrado por via parenteral. No entanto, como os GHS têm ação em nível hipotalâmico e hipofisário, só levariam ao aumento de GH e de IGF-1 em um grupo seleto de pacientes com deficiência de GH idiopática, que tenham deficiência funcional das vias estimuladoras de GH na hipófise, sem lesão anatômica, como no estudo descrito anteriormente (49).

$\mathrm{O}$ envelhecimento está associado à diminuição das concentrações séricas de GH e de IGF-l para aproximadamente metade das observadas na população adulta jovem, o que poderia contribuir para a redução de massa muscular e óssea e o aumento da adiposidade que ocorrem com a idade. O MK-0677 foi utilizado em pacientes idosos saudáveis e, após quatro semanas de uso, houve aumento significativo dos níveis de $\mathrm{GH}$ e de IGF-1, que atingiram valores normais para adultos jovens (53). Entretanto, seu uso não é bem estabelecido nesta população, de modo semelhante ao que ocor- re com o GH. Outra situação comum no envelhecimento é a anorexia, que leva à perda de peso involuntária e à desnutrição protéica, com aumento da morbimortalidade. Um recente estudo experimental (54) mostrou que ratos idosos que receberam ghrelina após jejum de 72 horas tiveram maior ingesta alimentar e maior recuperação do peso corporal, com predomínio de massa magra, comparados aos que não receberam ghrelina. Além disso, nesse mesmo estudo, os ratos tratados com ghrelina da $50^{\mathrm{a}}$ à $80^{\mathrm{a}}$ semana de vida tiveram aumento de peso similar aos não-tratados, porém com adiposidade significativamente menor, e sem diferenças no perfil glicêmico ou lipídico. Esses dados sugerem que os GHS/ghrelina podem ser candidatos promissores para o tratamento da anorexia e das mudanças metabólicas que ocorrem com a idade.

Sabe-se que o GH também estimula o turnover ósseo e aumenta a atividade osteoblástica. Assim, a associação de um agonista dos GHS a um inibidor de reabsorção óssea poderia aumentar a taxa de formação óssea na osteoporose. Um estudo com 292 mulheres com osteoporose, após a menopausa, mostrou que a associação do MK-0677 com o alendronato por 18 meses promoveu maior aumento de massa óssea no colo de fềmur que o uso exclusivo do alendronato (55). Não houve aumento de densidade óssea na coluna lombar e corpo total, mas o período de 18 meses é curto para avaliar os efeitos ótimos do estímulo do eixo $\mathrm{GH} /$ IGF-1 na formação óssea, já que foi demonstrado que a administração de $\mathrm{GH}$ em pacientes com deficiência de GH aumenta a densidade óssea somente após dois anos de uso (56).

O uso terapêutico dos análogos de GHS nos estados catabólicos também tem sido estudado. A administração do MK-0677 em homens jovens saudáveis submetidos à restrição calórica com balanço nitrogenado negativo promove elevação persistente de $\mathrm{GH}$ e de IGF-1, com reversão da perda de nitrogênio (57). Um outro agonista oral, o RC-1291, leva ao aumento do peso corporal após 8 a 11 dias de tratamento em indivíduos saudáveis (58). Quando administra-se ghrelina por infusão contínua em pacientes com anorexia causada por câncer, há aumento de $30 \%$ na ingestão alimentar e no apetite (59). Esses achados sugerem que os GHS e/ou a ghrelina podem melhorar os estados de catabolismo por meio da elevação dos níveis circulantes de GH e IGF-1, como também por mecanismos GHindependentes, como o aumento do apetite, o aumento do peso corporal e a inibição de citoquinas com 
efeito anorético (59). Assim, os GHS/ghrelina podem ser úteis no tratamento das síndromes consumptivas relacionadas ao câncer, ao HIV e a doenças crônicas.

\section{CONCLUSÃo}

A ghrelina é um novo hormônio, secretado do estômago para a circulação. Todavia, a ghrelina também é produzida no hipotálamo e em outros tecidos, com efeitos endócrinos e parácrinos. A sua modificação acil é essencial para os seus efeitos biológicos, isto é, aumento da liberação de GH e da ingestão alimentar. A contribuição da ghrelina circulante e da ghrelina hipotalâmica na secreção de GH ainda não está totalmente estabelecida. Os dados disponíveis sugerem que a ghrelina poderia ter papel fisiológico na secreção pulsátil de $\mathrm{GH}$, mas estudos adicionais são necessários para elucidar seu mecanismo de modulação da liberação de GH. Os agonistas sintéticos do GHS têm múltiplos potenciais terapêuticos, não somente na deficiência de $\mathrm{GH}$, mas em situações de alteração da composição corporal e do balanço energético.

\section{AGRADECIMENTOS}

As autoras agradecem aos pós-graduandos que contribuíram para este estudo, ao Prof. Dr. José G. H. Vieira pelo apoio laboratorial e à Aparecida Filomena Machado e à Walkiria Miranda pelo apoio com os ensaios.

\section{REFERÊNCIAS}

1. Bowers CY, Momany FA, Reynolds GA, Hong A. On the in vitro and in vivo activity of a new synthetic hexapeptide that acts on the pituitary to specifically release growth hormone. Endocrinology. 1984;114:1537-45.

2. Smith RG, Van der Ploeg LHT, Howard AD, Feighner SD, Cheng K, Hickey GJ, et al. Peptidomimetic regulation of growth hormone secretion. Endocr Rev. 1997;18:621-45.

3. Korbonits M, Grossman A. Growth hormone-releasing peptide and its analogues -novel stimuli to growth hormone release. Trends Endocrinol Metab. 1995;6:43-9.

4. Howard AD, Feighner SD, Cully DF, Arena JP, Liberator PA, Rosenblum $\mathrm{Cl}$, et al. A receptor in pituitary and hypothalamus that functions in growth hormone release. Science. 1996;273:947-77.

5. Kojima M, Hosoda H, Date Y, Nakazato M, Matsuo H, Kangawa K. Ghrelin is a growth-hormone-releasing acylated peptide from stomach. Nature. 1999; 402:656-60.

6. Arvat E, DiVito L, Broglio F, Papotti M, Muccioli G, Dieguez C, et al. Preliminary evidence that ghrelin, the natural GH secre- tagogue (GHS)-receptor ligand, strongly stimulates GH secretion in humans. J Endocrinol Invest. 2000;23:493-5.

7. Korbonits $M$, Goldstone AP, Gueorguiev M, Grossman AB. Ghrelin - a hormone with multiple functions. Front Neuroendocrinol. 2004;25:27-68.

8. Van der Lely AJ, Tschop M, Heiman ML, Ghigo E. Biological, physiological, pathophysiological, and pharmacological aspects of ghrelin. Endocr Rev. 2004;25:426-57.

9. Sun $Y$, Wang $P$, Zheng $H$, Smith RG. Ghrelin stimulation of growth hormone release and appetite is mediated through the growth hormone secretagogue receptor. Proc Natl Acad Sci U S A. 2004;101:4679-84.

10. Gnanapavan S, Kola B, Bustin SA, Morris DG, McGee P, Fairclough $P$, et al. The tissue distribution of the mRNA of ghrelin and subtypes of its receptor, GHS-R, in humans. J Clin Endocrinol Metab. 2002;87:2988-91.

11. Cummings DE, Weigle DS, Frayo RS, Breen PA, Ma MK, Dellinger EP, Purnell JQ. Plasma ghrelin levels after diet-induced weight loss or gastric bypass surgery. N Engl J Med. 2002;346:1623-30.

12. Banks WA, Tschop M, Robinson SM, Heiman ML. Extent and direction of ghrelin transport across the blood-brain barrier is determined by its unique primary structure. J Pharmacol Exp Ther. 2002;302:822-7.

13. Takaya K, Ariyasu $H$, Kanamoto $N$, Iwakura $H$, Yoshimoto $A$, Harada M, et al. Ghrelin strongly stimulates growth hormone release in humans. J Clin Endocrinol Metab. 2000;85:4908-11.

14. Arvat E, Maccario M, DiVito L, Broglio F, Benso A, Gottero C, et al. Endocrine activities of ghrelin, a natural growth hormone secretagogue (GHS), in humans: comparison and interactions with hexarelin, a nonnatural peptidyl GHS, and GH-releasing hormone. J Clin Endocrinol Metab. 2001;86:1169-74.

15. Peñalva $A$, Carballo $A$, Pombo $M$, Casanueva FF, Dieguez $C$. Effect of growth hormone (GH)-releasing hormone (GHRH), atropine, pyridostigmine, or hypoglycemia on GHRP-6-induced GH secretion in man. J Clin Endocrinol Metab. 1993;76:168-71.

16. Hataya Y, Akamizu T, Takaya K, Kanamoto N, Ariyasu H, Saijo $\mathrm{M}$, et al. A low dose of ghrelin stimulates growth hormone $\mathrm{GH})$ release synergistically with $\mathrm{GH}$-releasing hormone in humans. J Clin Endocrinol Metab. 2001;86:4552-5.

17. Popovic V, Damjanovic S, Micic D, Djurovic M, Dieguez C, Casanueva FF. Blocked growth hormone-releasing peptide (GHRP-6)-induced GH secretion and absence of the synergic action of GHRP-6 plus GH-releasing hormone in patients with hypothalamopituitary disconnection: evidence that GHRP-6 main action is exerted at the hypothalamic level. J Clin Endocrinol Metab. 1995;80:942-7.

18. Popovic V, Miljic D, Micic D, Damjanovic S, Arvat E, Ghigo E, et al. Ghrelin main action on the regulation of growth hormone release is exerted at hypothalamic level. J Clin Endocrinol Metab. 2003;88:3450-3.

19. Tannenbaum GS, Bowers CY. Interactions of growth hormone secretagogues and growth hormone-releasing hormone/somatostatin. Endocrine. 2001;14:21-7.

20. Pandya N, Mott-Friberg R, Bowers CY, Barkan AL, Jaffe CA. Growth hormone $(\mathrm{GH})$-releasing peptide-6 requires endogenous hypothalamic GH-releasing hormone for maximal GH stimulation. J Clin Endocrinol Metab. 1998;83:1186-9.

21. Dickson SL, Doutrelant-Viltart O, Leng G. GH-deficient dw/dw rats and lit/lit mice show increased Fos expression in the hypothalamic arcuate nucleus following systemic injection of GH-releasing peptide-6. J Endocrinol. 1995;146:519-26.

22. Maheshwari H, Rahim A, Shalet SM, Baumann G. Selective lack of growth hormone $(\mathrm{GH})$ response to the $\mathrm{GH}$-releasing 
peptide hexarelin in patients with $\mathrm{GH}$-releasing hormone receptor deficiency. J Clin Endocrinol Metab. 1999;84:956-9.

23. Wren AM, Small CJ, Fribbens CV, Neary NM, Ward HL, Seal LJ, et al. The hypothalamic mechanisms of the hypophysiotropic action of ghrelin. Neuroendocrinology. 2002;76:316-24.

24. Korbonits M, Little JA, Forsling ML, Tringali G, Costa A, Navarra $\mathrm{P}$, et al. The effect of growth hormone secretagogues and neuropeptide $Y$ on hypothalamic hormone release from acute rat hypothalamic explants. J Neuroendocrinol. 1999;11:521-8.

25. Guillaume V, Magnan E, Cataldi M, Dutour A, Sauze N, Renard $\mathrm{M}$, et al. Growth hormone (GH)-releasing hormone secretion is stimulated by a new GH-releasing hexapeptide in sheep. Endocrinology. 1994;135:1073-6.

26. Goth MI, Lyons CE, Canny BJ, Thorner MO. Pituitary adenylate cyclase activacting polypeptide, growth hormone (GH)-releasing peptide and $\mathrm{GH}$-releasing hormone stimulate $\mathrm{GH}$ release through distinct pituitary receptors. Endocrinology. 1992;130:939-44.

27. Malagon MM, Luque RM, Ruiz-Guerrero E, Rodríguez-Pacheco F, García-Navarro S, Casanueva FF, et al. Intracellular signaling mechanisms mediating ghrelin-stimulated growth hormone release in somatotropes. Endocrinology. 2003;144:5372-80.

28. Kineman RD, Luque RM. Evidence that ghrelin is as potent as growth hormone (GH)- releasing hormone (GHRH) in releasing GH from primary pituitary cell cultures of a nonhuman primate (Papio anubis), acting through intracellular signaling pathways distinct from GHRH. Endocrinology. 2007; 148:4440-9.

29. Tolle V, Bassant MH, Zizzari P, Poindessous-Jazat F, Tomasetto C, Epelbaum J, Bluet-Pajot MT. Ultradian rhythmicity of ghrelin secretion in relation with $\mathrm{GH}$, feeding behavior, and sleepwake patterns in rats. Endocrinology. 2002;143:1353-61.

30. Okimura $Y$, Ukai K, Hosoda H, Murata M, Iguchi G, lida K, et al. The role of circulating ghrelin in growth hormone $(\mathrm{GH})$ secretion in freely moving male rats. Life Sci. 2003;72:2517-24.

31. Tannenbaum GS, Epelbaum J, Bowers CY. Interrelationship between the novel peptide ghrelin and somatostatin/growth hormone-releasing hormone in regulation of pulsatile growth hormone secretion. Endocrinology. 2003;144:967-74.

32. Barkan AL, Dimaraki EV, Jessup SK, Symons KV, Ermolenko $M$, Jaffe CA. Ghrelin secretion in humans is sexually dimorphic, suppressed by somatostatin, and not affected by the ambient growth hormone levels. J Clin Endocrinol Metab. 2003;88:2180-4.

33. Halem HA, Taylor JE, Dong JZ, Shen Y, Datta R, Abizaid A, et al. Novel analogs of ghrelin: physiological and clinical implications. Eur J Endocrinol. 2004;151 (Suppl 1):S71-5.

34. Zizzari P, Halem H,Taylor J, Dong JZ, Datta R, Culler MD, et al. Endogenous ghrelin regulates episodic growth hormone (GH) secretion by amplifying GH pulse amplitude: evidence from antagonism of the GH secretagogue-R1a receptor. Endocrinology. 2005; 146:3836-42.

35. Tannenbaum GS, Samia M, Chen Q, et al. Antagonism of the growth hormone secretagogue receptor unmasks a role for ghrelin in maintaining high GH pulse amplitude under physiological conditions. Endo Soc 87th Ann Meet 2005; San Diego, USA, June 4-7.

36. Pantel J, Legendre M, Cabrol S, Hilal L, Hajaji Y, Morisset S, et al. Loss of constitutive activity of the growth hormone secretagogue receptor in familial short stature. J Clin Invest. 2006;116:760-8.

37. Koutkia P, Canavan B, Breu J, Johnson ML, Grinspoon SK. Nocturnal ghrelin pulsatility and response to growth hormone secretagogues in healthy men. Am J Physiol Endocrinol Metab. 2004;287:E506-12.

38. Kamegai J, Tamura H, Shimizu T, Ishii S, Tatsuguchi A, Sugiha$\mathrm{ra} \mathrm{H}$, et al. The role of pituitary ghrelin in growth hormone (GH) secretion: GH-releasing hormone-dependent regulation of pituitary ghrelin gene expression and peptide content. Endocrinology. 2004;145:3731-8.

39. Sun Y, Ahmed S, Smith RG. Deletion of ghrelin impairs neither growth nor appetite. Mol Cell Biol. 2003;23:7973-81.

40. Shuto $Y$, Shibasaki T, Otagiri A, Kuriyama H, Ohata $H$, Tamura $\mathrm{H}$, et al. Hypothalamic growth hormone secretagogue receptor regulates growth hormone secretion, feeding, and adiposity. J Clin Invest. 2002;109:1429-36.

41. Peino R, Baldelli R, Rodriguez-Garcia J, Rodriguez-Segade S, Kojima M, Kangawa K, et al. Ghrelin-induced growth hormone secretion in humans. Eur J Endocrinol. 2000;143:11-4.

42. Broglio F, Benso A, Castiglioni C, Gottero C, Prodam F, Destefanis $S$, et al. The endocrine response to ghrelin as a function of gender in humans in young and elderly subjects. J Clin Endocrinol Metab. 2003;88:1537-42.

43. Cordido F, Penalva A, Dieguez C, Casanueva FF. Massive growth hormone $(\mathrm{GH})$ discharge in obese subjects after the combined administration of GH-releasing hormone and GHRP-6: evidence for a marked somatotroph secretory capability in obesity. J Clin Endocrinol Metab. 1993;76:819-23.

44. Tassone F, Broglio F, Destefanis S, Rovere S, Benso A, Gottero $C$, et al. Neuroendocrine and metabolic effects of acute ghrelin administration in human obesity. J Clin Endocrinol Metab. 2003;88:5478-83.

45. Maccario M, Arvat E, Procopio M, Gianotti L, Grottoli S, Imbimbo BP, et al. Metabolic modulation of the growth hormone-releasing activity of hexarelin in man. Metabolism. 1995;44:134-8.

46. Di Vito L, Broglio F, Benso A, Gottero C, Prodam F, Papotti M, et al. The GH-releasing effect of ghrelin, a natural GH secretagogue, is only blunted by the infusion of exogenous somatostatin in humans. Clin Endocrinol (Oxf). 2002;56:643-8.

47. Lengyel AM. From growth hormome- releasing peptides to ghrelin: discovery of new modulators of GH secretion. Arq Bras Endocrinol Metab. 2006;50:17-24.

48. Lengyel AM. Novel mechanisms of growth hormone regulation: growth hormone-releasing peptides and ghrelin. Braz J Med Biol Res. 2006;39:1003-11.

49. Chapman IM, Pescovitz OH, Murphy G, Treep T, Cerchio KA, Krupa D, et al. Oral administration of growth hormone (GH) releasing peptide-mimetic MK-677 stimulates the $\mathrm{GH} /$ insulinlike growth factor-I axis in selected GH-deficient adults. J Clin Endocrinol Metab. 1997;82:3455-63.

50. Codner E, Cassorla F, Tiulpakov AN, Mericq MV, Avila A, Pescovitz $\mathrm{OH}$, et al. Effects of oral administration of ibutamoren mesylate, a nonpeptide growth hormone secretagogue, on the growth hormone-insulin-like growth factor I axis in growth hormone-deficient children. Clin Pharmacol Ther. 2001;70:91-8.

51. Svensson J, Monson JP, Vetter T, Hansen TK, Savine R, Kann P, et al. and NN703 Clinical Research Group. Oral administration of the growth hormone secretagogue NN703 in adult patients with growth hormone deficiency. Clin Endocrinol (Oxf). 2003;58:572-80.

52. Pihoker C, Badger TM, Reynolds GA, Bowers CY. Treatment effects of intranasal growth hormone releasing peptide-2 in children with short stature. J Endocrinol. 1997;155:79-86.

53. Chapman IM, Bach MA, Van Cauter E, Farmer M, Krupa D, Taylor AM, et al. Stimulation of the growth hormone (GH)-insulin-like growth factor I axis by daily oral administration of a 
GH secretogogue (MK-677) in healthy elderly subjects. J Clin Endocrinol Metab. 1996;81:4249-57.

54. Ariyasu H, Iwakura H, Yamada G, Nakao K, Kangawa K, Akamizu T. Efficacy of Ghrelin as a therapeutic approach for age-related physiologic changes. Endocrinology [serial on the Internet] 2008 27. [Epub]

55. Murphy MG, Weiss S, McClung M, Schnitzer T, Cerchio K, Connor J, et al. and MK-677/Alendronate Study Group. Effect of alendronate and MK-677 (a growth hormone secretagogue), individually and in combination, on markers of bone turnover and bone mineral density in postmenopausal osteoporotic women. J Clin Endocrinol Metab. 2001;86:1116-25.

56. Gillberg P, Mallmin H, Petrén-Mallmin M, Ljunghall S, Nilsson AG. Two years of treatment with recombinant human growth hormone increases bone mineral density in men with idiopathic osteoporosis. J Clin Endocrinol Metab. 2002;87:4900-6.

57. Murphy MG, Plunkett LM, Gertz BJ, He W, Wittreich J, Polvino WM, Clemmons DR. MK-677, an orally active growth hormone secretagogue, reverses diet-induced catabolism. J Clin Endocrinol Metab. 1998;83:320-5.
58. Garcia JM, Polvino WJ. Effect on body weight and safety of RC-1291, a novel, orally available ghrelin mimetic and growth hormone secretagogue: results of a phase I, randomized, placebo-controlled, multiple-dose study in healthy volunteers. Oncologist. 2007;12:594-600.

59. Neary NM, Small CJ, Wren AM, Lee JL, Druce MR, Palmieri C, et al. Ghrelin increases energy intake in cancer patients with impaired appetite: acute, randomized, placebo-controlled trial. J Clin Endocrinol Metab. 2004;89:2832-6.

Endereço para correspondência:

Silvia R. Correa-Silva

Disciplina de Endocrinologia, EPM/Unifesp

Rua Pedro de Toledo, 910

04039-002 São Paulo, SP

E-mail: dra.silviaregina@terra.com.br 